\title{
Self-assembled nanostructured photonic-plasmonic metasurfaces for high-resolution optical thermometry
}

\author{
Giorgio Baraldi, Marina García Pardo, José Gonzalo, Rosalia Serna*, Johann Toudert*
}

Dr. G. Baraldi, M. García Pardo, Dr. J. Gonzalo, Prof. R. Serna, Dr. J. Toudert

Laser Processing Group, Instituto de Óptica, IO, CSIC, Madrid, Spain

E-mail: johann.toudert@gmail.com,rosalia.serna@csic.es

Keywords: Metasurfaces, Self-assembly, Plasmon, Optical phase, Remote sensing

Abstract. Sensing devices for environment, safety, healthcare, and optoelectronic

applications require an accurate and non-invasive monitoring of their temperature, because its variations markedly affect the overall response of the device. Monitoring the optical response of temperature-sensitive integrated photonic elements, such as micro-resonators or microinterferometers, is an appealing solution in this context. However, achieving high-resolution optical thermometry with such elements is unpractical and costly as this requires lithography processing, highly monochromatic laser sources, complex light coupling strategies. Here, a photonic-plasmonic metasurface design that enables practical optical thermometry with a sub$10^{-3}{ }^{\circ} \mathrm{C}$ resolution is proposed. It is based on a self-assembled nanostructured material implemented with a lithography-free process. The optical response of the temperaturesensitive metasurface is probed using a white light source and by monitoring the optical phase in a standard reflectance configuration. This facile, yet powerful, sensing scheme stands on the effective response of the metasurface, which involves the hybridization of thin film interference and low-quality-factor plasmon resonances to enable a quasi-darkness response with a sharp spectral variation (jump) of the optical phase. Such jump is equivalent with a high-quality-factor resonator that yields a high sensor responsivity and thus enables highresolution optical thermometry. 
Main Text. Optical monitoring is a powerful diagnostic and control tool needed for remote and non-invasive sensing applications in the environment, safety, healthcare, or optoelectronics areas. For such applications that aim at detecting low levels of gases, particles, biomolecules or light, it is first primordial to monitor accurately temperature because its variations affect the overall response of the sensing device. Therefore, it will be desirable for the next generation of such devices to integrate, in microscale or smaller dimensions, highresolution optical thermometry functionalities, i.e. the capability of monitoring with light very slight changes in their temperature.

In this context, monitoring the optical response of temperature-sensitive integrated photonic elements, such as micro-resonators ${ }^{[1-8]}$ or micro-interferometers ${ }^{[9-10]}$ has arisen as an appealing solution. With such elements, temperature monitoring requires tracking the wavelength variation of narrow, high-quality-factor resonances (spectral width at the picometer scale). The reported responsivities reaching up to $200 \mathrm{pm} /{ }^{\circ} \mathrm{C}^{[7-10]}$ indicate a higher resolution than other implementations, such as localized surface plasmon resonators, ${ }^{[11,12]}$ surface plasmon polariton resonators, ${ }^{[13-15]}$ Bragg gratings ${ }^{[16]}$ or Tamm structures. ${ }^{[17]}$ Yet, although record resolutions down to the $10^{-9}{ }^{\circ} \mathrm{C}$ range ${ }^{[6]}$ have been obtained with micro-resonators, the reported responsivities imply that achieving even a $10^{-3}{ }^{\circ} \mathrm{C}$ resolution requires a picometer precision in the determination of the resonance wavelength. Reaching such precision requires specific devices, such as highly monochromatic lasers as light source or bulky optoelectronic devices for signal processing. Furthermore, micro-resonators and micro-interferometers suffer from their weak interaction with the incident light. Therefore, their use requires employing high optical powers or designing additional and specific in-coupling structures. The fabrication of such structures together with the micro-resonators and micro-interferometers 
require high-accuracy lithography setups. In sum, although such photonic elements permit record high-resolution optical thermometry, they remain an unpractical and costly solution.

A promising and yet unexplored alternative for practical and cost-effective high-resolution optical thermometry consists in monitoring the optical response of temperature-sensitive metasurfaces. Metasurfaces ${ }^{[18-20]}$ can support a rich variety of optical modes that enable a straightforward strong coupling of light in their subwavelength thickness. Furthermore, their optical response can be made broadly tunable through changes in their environment, enabling a dynamic shaping of the amplitude and phase of light. ${ }^{[21-35]}$ Such tunability also opens the way to using metasurfaces as "wireless" sensors allowing an optical monitoring of their environment. Sensing with particularly high resolution has been demonstrated by monitoring the environment-sensitive optical response of metasurfaces presenting topological darkness. ${ }^{[36,37]}$ Topological darkness is characterized by a zero amplitude and a phase singularity for the Fresnel reflection coefficient of the metasurface occurring at specific photon energies and angles of incidence. Although initially shown for linearly p-polarized light, topological darkness can also occur with other polarization states. ${ }^{[37-40]}$ By slightly detuning the angle of incidence from topological darkness conditions, a small fraction of light is reflected by the metasurface and the phase of this light varies abruptly (or "jumps") with photon energy while remaining defined. In the spectral region of this jump, the reflected light phase is by far more sensitive to any change in the optical response of the environmentsensitive metasurface than the reflected light intensity. For this reason, monitoring the phase of light reflected by metasurfaces in quasi-darkness conditions enabled sensing with a particularly high resolution. This was successfully applied for the optical detection of very low levels of hydrogen and biomarkers using plasmonic metasurfaces as sensing platform. ${ }^{[36,37]}$ Following a similar scheme, recent works have reported a high-resolution 
sensing potential for other material architectures such as $3 \mathrm{D}$ metamaterials, ${ }^{[40-42]}$ surface plasmon polariton resonators ${ }^{[43]}$ or Tamm plasmonic structures. ${ }^{[44]}$

Here, we report a temperature-sensitive metasurface that enables practical high-resolution optical thermometry, based on the monitoring of its reflected light phase in quasi-darkness conditions. Such metasurface, which consists of a self-assembled photonic-plasmonic structure, enables a temperature resolution below the $10^{-3}{ }^{\circ} \mathrm{C}$ range using a standard optical reflectance measurement configuration and a white light source. This facile and yet powerful experimental scheme for sensing is enabled by the effective response of the metasurface that involves the hybridization of thin film interference ("photonic") and low-quality-factor plasmon resonances ("plasmonic"), which together in the quasi-darkness conditions behave, in the optical phase space, like a high-quality-factor resonator that enables a high sensing resolution. These results show the potential of self-assembled photonic-plasmonic metasurfaces as a practical alternative to micro-resonators and micro-interferometers for temperature sensing. Such class of metasurface can be fabricated without expensive lithography tools and enables a high resolution in easy-to-implement and cost-effective optical measurement conditions.

Nanostructured metasurfaces were fabricated by pulsed laser deposition. A silver (Ag) coating was first deposited on silicon substrates covered with a thin layer of amorphous aluminium oxide $\left(\mathrm{a}-\mathrm{Al}_{2} \mathrm{O}_{3}\right)$. Then this coating was annealed at $350^{\circ} \mathrm{C}$ to form a well-defined $2 \mathrm{D}$ array of self-assembled $\mathrm{Ag}$ nanoparticles. Finally, an a- $\mathrm{Al}_{2} \mathrm{O}_{3}$ cover layer was deposited onto the $\mathrm{Ag}$ nanoparticles. Figure 1a depicts the metasurface structure before and after deposition of this cover layer ("uncover" and "cover", respectively). The "uncover" metasurface consists of an amorphous 2D array of Ag nanoparticles with a hemispherical shape and an average in-plane diameter near $100 \mathrm{~nm}$. The top surface of these nanoparticles is in contact with air. In the 
"cover" metasurface, the Ag nanoparticles present a distribution, hemispherical shape and average diameter similar to those of the "uncover" metasurface. However, in this case, the nanoparticles are fully buried below the $\mathrm{a}-\mathrm{Al}_{2} \mathrm{O}_{3}$ cover layer, whose topography replicates conformally the morphology and distribution of the nanoparticles underneath. The top of the "cover" metasurface thus consists of an amorphous 2D array of nanodomes on a flat background (Supporting Information S1).

The optical properties in the ultraviolet, visible and near infrared of the "uncover" and “cover" metasurfaces were first characterized by specular reflectance spectroscopy with sand p- polarized light, in a broad range of angles of incidence (AOI from $20^{\circ}$ to $70^{\circ}$ ), at room temperature. The measurement configuration is depicted in Figure 1b, and the setup is described in Supporting Information S2. For the "cover" metasurface (Figure 1c (bottom panel)), the p-polarized reflectance $\mathrm{R}_{\mathrm{p}}$ presents a marked minimum with a near-zero value $\left(\mathrm{R}_{\mathrm{p}}{ }^{\min } \approx 0.25^{\circ}\right)$ at the $\mathrm{AOI}$ of $48^{\circ}$ for a photon energy $\mathrm{E} \approx 2.8 \mathrm{eV}$. This response is characteristic of quasi-darkness conditions, where the p-polarized beam is very weakly reflected by the metasurface. No such conditions could be observed at any AOI and polarization ( $\mathrm{s}$ or $\mathrm{p}$ ) for the "uncover" metasurface. This can be seen from Figure 1c (top panel) that shows the reflectance spectra of this structure at the AOI of $65^{\circ}$, at which its most pronounced minima were observed for $\mathrm{R}_{\mathrm{p}}$. These minima are not marked enough $\left(\mathrm{R}_{\mathrm{p}}{ }^{\mathrm{min}} \approx 4^{\circ}\right.$ and $14^{\circ}$ ) to allow quasi-darkness conditions to be fulfilled.

Spectroscopic ellipsometry measurements in the ultraviolet, visible and near infrared were then performed on the two kinds of metasurfaces to determine the amplitude and phase of the reflected light, at room temperature. Details about the measurement setup are given in Supporting Information S2. Figure 1d shows the spectra of the ellipsometric amplitude 
coefficient $\Psi$ of the "uncover" (top panel) and "cover" (bottom panel) metasurfaces. This coefficient represents the $\left|r_{p} / r_{s}\right|$ ratio, $r_{p}$ and $r_{s}$ being the Fresnel reflection coefficients of the metasurface, which relate with reflectance following the relations: $R_{p}=\left|r_{p}\right|^{2} ; R_{s}=\left|r_{s}\right|^{2}$. Therefore, quasi-darkness conditions for p-polarized light $\left(\mathrm{R}_{\mathrm{p}} \rightarrow 0\right)$ translate into a sharp minimum and a near cancelation of $\Psi$ as observed for the "cover" metasurface for AOI $=48^{\circ}$ and $\mathrm{E} \approx 2.8 \mathrm{eV}$. No other sharp minimum of $\Psi$ could be observed in the studied spectral range for this metasurface, even by varying the AOI (Supporting Information S2, Figure S2.1.).

Note however that sharp $\Psi$ maxima are observed at $\left(\mathrm{AOI}=60^{\circ}, \mathrm{E} \approx 2.65 \mathrm{eV}\right)$ and $\left(\mathrm{AOI}=70^{\circ}\right.$, $\mathrm{E} \approx 3.2 \mathrm{eV}$ ), due to the near-zero value of $\mathrm{R}_{\mathrm{s}}$ in these conditions for which the "cover" metasurface presents quasi-darkness for s-polarized light. As no such near-zero values of neither $\mathrm{R}_{\mathrm{s}}$ nor $\mathrm{R}_{\mathrm{p}}$ are achieved at any AOI for the "uncover" metasurface, its $\Psi$ spectra depend weakly on photon energy (Supporting Information S2, Figure S2.2).

The spectrum of the ellipsometric phase coefficient $\Delta$ of the "cover" metasurface for an AOI of $48^{\circ}$ is shown in Figure 2a (bottom panel). This coefficient represents the phase shift between the $\mathrm{p}$ - and s-polarized components of light induced by reflection. At $\mathrm{E}=2.8 \mathrm{eV}$ where quasi-darkness conditions are fulfilled for p-polarized light $\left(\mathrm{R}_{\mathrm{p}} \rightarrow 0\right.$ and $\Psi \rightarrow$ $0), \Delta$ turns abruptly by $170^{\circ}$, from $260^{\circ}$ to $90^{\circ}$. This behaviour originates from a jump in the phase of the near-zero-intensity p-polarized reflected light. ${ }^{[37]}$ Note that additional abrupt $\Delta$ variations can be seen at the AOIs and photon energies of the sharp $\Psi$ maxima where $\mathrm{R}_{\mathrm{s}} \rightarrow$ 0 (Supporting Information S2, Figure S2.1). In that case, such variations originate from jumps in the phase of the near-zero-intensity s-polarized reflected light.

To elucidate the origin of the quasi-darkness conditions and related phase jump in the "cover" metasurface, we modeled it as a layered structure (Figure 2b) in which the buried Ag 
nanoparticles layer is represented by an effective medium and the conformal a- $\mathrm{Al}_{2} \mathrm{O}_{3}$ cover layer is represented by a graded medium. More details about the model and simulations are given in Supporting Information S3. In particular, we show in the Supporting Information S3 that the effective medium approach is suitable for fitting the experimental $\Psi, \Delta, \mathrm{R}_{\mathrm{p}}$ and $\mathrm{R}_{\mathrm{s}}$ spectra of the "cover" metasurface in a broad range of AOIs, from $20^{\circ}$ to $70^{\circ}$. This supports the validity of the proposed effective medium-based modeling for describing the macroscopic optical properties of this specific metasurface that are concerned in the sensing experiment, and for providing an equivalent model capturing its relevant physical features and describing its sensing performance. From numerical fitting of the experimental $\Psi$ and $\Delta$ spectra, the effective refractive index $n_{\text {eff }}$ and extinction coefficient $k_{\text {eff }}$ of the buried Ag nanoparticles layer were determined. Their spectra, shown in Figure 2c, are dominated by two resonances, peaking near $2.2 \mathrm{eV}$ and $2.9 \mathrm{eV}$, in good qualitative agreement with the transmittance spectrum of a similar "cover" metasurface grown on a transparent fused silica substrate (Supporting Information S4). Both resonances are also seen in the transmittance spectrum of an "uncover" metasurface on fused silica, where they nevertheless peak at higher photon energies (Supporting Information S4). These environment-sensitive resonances are attributed to the localized surface plasmon resonance modes of the Ag nanoparticles: dipolar and quadrupolar modes near $2.2 \mathrm{eV}$ and $2.9 \mathrm{eV}$, respectively. For the "cover" metasurface, these resonances enable $n_{\text {eff }}$ and $k_{\text {eff }}$ values suitable for achieving almost fully destructive interference for p-polarized light in the layered structure at a specific AOI $\left(48^{\circ}\right)$ and photon energy $(\mathrm{E} \approx 2.8 \mathrm{eV}$ ) (Figure $2 \mathrm{~b}$ and Supporting Information S5). Therefore, the quasidarkness conditions of the "cover" metasurface, and thus the related phase jump, can be described with an equivalent model in which the interplay between thin film interference and localized surface plasmon resonances confers them a hybrid origin. Such equivalent model of the "cover" metasurface reflects the photonic-plasmonic design of the "cover" metasurface. 
According to this model, the magnitude and photon energy of the plasmon resonances are primarily defined by the Ag nanoparticles size, shape, 2D organization and environment, and the tuning of the vertical layered structure enables the necessary phase shifts to achieve fully destructive interference at the required photon energies and AOIs. Thus, it is important to note the key role of the a- $\mathrm{Al}_{2} \mathrm{O}_{3}$ cover layer in the design of the metasurface: according to the proposed equivalent model, the absence of an $\mathrm{a}-\mathrm{Al}_{2} \mathrm{O}_{3}$ cover layer limits the optical path in the layered structure, in which the accumulated phase shifts are insufficient to achieve fully destructive interference at any AOI. This simple theoretical consideration is validated by the fact that no quasi-darkness and phase jumps are observed for the "uncover" metasurface (Figure S2.2), thus further showing the capability of the proposed equivalent model to capture the relevant physical features of the studied metasurfaces.

Here, we show the suitability of the "cover" metasurface as temperature-sensitive platform for optical thermometry, by monitoring the phase of the reflected p-polarized light in quasidarkness conditions. To demonstrate this property, the $\Delta$ spectrum of this metasurface was measured by spectroscopic ellipsometry as a function of temperature in the $25^{\circ} \mathrm{C}$ to $55^{\circ} \mathrm{C}$ range, for an AOI of $48^{\circ}$. Figure $3 \mathrm{a}$ shows the measured spectra in the region near the phase jump, at selected temperatures. Upon temperature increase from $25^{\circ} \mathrm{C}$ to $45^{\circ} \mathrm{C}, \Delta$ varies gradually in a narrow spectral region $(2.806-2.808 \mathrm{eV}$, or $441.5-441.2 \mathrm{~nm})$ where a $180^{\circ}$ change is finally achieved. Upon increasing temperature to $55^{\circ} \mathrm{C}$, the $\Delta$ jump flips completely. Furthermore, as shown in Figure $3 b$, the specular reflectance $R_{p}$ and $R_{s}$ values of the metasurface remain unchanged upon varying temperature in the range considered here. Thus, probing the phase of the reflected light in the spectral region of the jump allows the monitoring of temperature with a much higher resolution than with traditional specular reflectance measurements. 
To estimate the achievable resolution with such optical thermometry scheme, the ellipsometric phase coefficient $\Delta$ has been plotted as a function of temperature, at a selected photon energy $(E=2.807 \mathrm{eV}$, Figure $3 \mathrm{c})$. At this photon energy, the $\Delta=\mathrm{f}($ temperature $)$ curve shows a maximum slope at the low temperature side of the graph $\left(25^{\circ} \mathrm{C}\right)$. A linear fitting in this region yields a slope of $15^{\circ}$ in $\Delta$ per $1^{\circ} \mathrm{C}$ in temperature. Thus, with a $0.1^{\circ}$ resolution in the $\Delta$ measurement, the temperature will be determined with a $7 \times 10^{-3}{ }^{\circ} \mathrm{C}$ resolution. With a $0.01^{\circ}$ resolution in the $\Delta$ measurement (standard limit in ellipsometry experiments), temperature can be determined with a $7 \times 10^{-4}{ }^{\circ} \mathrm{C}$ resolution. Note that because the $\Delta$ measurements are performed in quasi-darkness conditions, the measured optical signals present a very low intensity. Therefore, reaching the temperature resolutions given above requires to maximize the signal-to-noise ratio in the measurement of $\Delta$ (by realizing, for instance, noise filtering, averaging, mesurement at different photon energies and spectral fitting). By plotting $\Delta=\mathrm{f}$ (temperature) at a different photon energy (Supporting Information S6, Figure S6.2), the temperature range of optimum resolution can be shifted to higher temperatures. Therefore, it is possible to achieve optical thermometry with high resolution in a relatively broad temperature range by simply monitoring the phase of the reflected light at a different photon energy. To further evaluate the potential of the "cover" metasurface for optical thermometry, its stability versus heating-cooling cycles was tested, showing a good reversibility upon cycling between $25^{\circ} \mathrm{C}$ and $55^{\circ} \mathrm{C}$. The proof of such reversibility is shown in the Supporting Information S6, Figure S6.3, where it is shown that the final $\Delta$ spectrum of the metasurface after $25^{\circ} \mathrm{C} \rightarrow 55^{\circ} \mathrm{C} \rightarrow 25^{\circ} \mathrm{C}$ heating-cooling is identical to the initial one. Such reversibility is likely favorized by the stability and robustness of the a- $\mathrm{Al}_{2} \mathrm{O}_{3}$ cover layer, which is very efficient in blocking the temperature-induced reshaping of metal nanoparticles, their chemical degradation, and material exo-diffusion. ${ }^{[45,46]}$ To demonstrate the temperature 
sensitivity of a "cover" metasurface in a real experiment; we studied the evolution of its $\Delta$ upon laser heating, where a continuous laser beam was shined onto the metasurface. The results of such experiment are shown on Figure 3d. Upon turning on the laser beam, the $\Delta$ jump shifts towards a smaller photon energy, as a result of the laser-induced temperature increase. After turning off the laser, the $\Delta$ jump comes back to its initial position. This points again at the good reversibility of the "cover" metasurface.

Summarizing, we have unveiled a non-demanding photonic-plasmonic metasurface design suitable for being used as temperature-sensitive platform for high-resolution optical thermometry. It is much easier to implement than previously reported photonic-plasmonic architectures that combined plasmonic nanostructures with micro-resonators or photonic structures to achieve highly environment-sensitive hybrid resonances with high quality factor and small mode volume. ${ }^{[47-51]}$ The on-chip implementation of such structures required using lithography techniques or building periodic multilayers thicker than the wavelength of light. Such fabrication routes are also needed for the implementation of micro-resonators, ${ }^{[1-8]}$ microinterferometers, ${ }^{[9,10]}$ or Tamm plasmonic structures, ${ }^{[17,44]}$ respectively. Here, the metasurface structure is compatible with a lithography-free two-layer fabrication process, affordable either by physical deposition or solution-processing. Because the size/shape/distance distribution of the Ag nanoparticles in the metasurface is dictated only by the initial Ag layer thickness and the Ag annealing temperature and atmosphere, which can be very accurately controlled together with the a- $\mathrm{Al}_{2} \mathrm{O}_{3}$ layer thicknesses during the pulsed laser deposition process (where thickness accuracy is less than $1 \mathrm{~nm}$ ), a good reproducibility can be achieved for the macroscopic optical properties of the metasurface that are probed during the sensing experiments. For instance, we estimate that, at best, a $10 \mathrm{~nm}$ reproducibility can be achieved for the plasmon resonance wavelength. Let us note that the proof-of-concept metasurface we 
show is simple and thus at reach of other well established self-assembly fabrication techniques.

The high sensing resolution enabled by such non-demanding design originates from the hybridization of two low-quality-factor phenomena, thin film interference and localized surface plasmon resonances, which yields quasi-darkness conditions where a sharp spectral variation ("a jump") of the phase of light reflected by the metasurface occurs. This jump is equivalent, for sensing purposes, with a high-quality-factor resonator showing a strong sensitivity to temperature. By monitoring the phase of light reflected by the temperaturesensitive metasurface in such quasi-darkness conditions, a temperature resolution below the $10^{-3}{ }^{\circ} \mathrm{C}$ range can be reached. This high resolution can be achieved in a broad range of temperature upon tuning the probe photon energy.

Finally, it is important that these high-resolution optical thermometry measurements have been realized in a standard optical reflectance configuration with no need of special light incoupling or out-coupling strategies or structures (Supporting Information S2). Furthermore, the measurements were done using a lamp by selecting a relatively broad light bandwidth ( 8 $\mathrm{nm}$, Supporting Information S2). Therefore, in contrast with micro-resonators or microinterferometers, neither a high light intensity, nor a highly monochromatic laser source are required for the proposed sensing scheme. Indeed, the spectral bandwidth does not affect strongly the phase jump (Supporting Information S7), and in fact it can be set as fixed parameter during the sensing experiment. This provides an advantage for the proposed sensing scheme versus other approaches based on active sensing systems, such as luminescence nanothermometry. Such approaches, which have shown excellent temperature resolutions (they enable reaching the range of $10^{-3}{ }^{\circ} \mathrm{C}$ ), ${ }^{[2,53]}$ however usually require the use of laser sources to induce luminescence signals with enough intensity. As a conclusion, this work shows how an easily built photonic-plasmonic metasurface design is suitable to enable high-resolution optical thermometry with an easy-to-implement 
measurement scheme. This points at its powerful potential as a practical alternative to microresonators and micro-interferometers for optical thermometry. Furthermore, the concept discussed here can be applied to a broader range of materials (beyond noble metals and common dielectrics) to enable higher temperature resolutions in ultrathin metasurfaces.

\section{Experimental Section}

The metasurfaces were fabricated by pulsed laser deposition in a vacuum chamber, where $\mathrm{Ag}$ and $\mathrm{Al}_{2} \mathrm{O}_{3}$ targets were ablated sequentially. The setup is described in detail in ref. 45 and references therein. The annealing at $350^{\circ} \mathrm{C}$ of the $\mathrm{Ag}$ coating was done in air. The specular reflectance and spectroscopic ellipsometry measurements were done with a VASE ellipsometer (Woollam Co. Inc.); more details about these measurements and the setup configurations are given in Supporting Information S2. The temperature-dependent ellipsometry measurements were done with the same setup, equipped with an Instec heat stage. These measurements were done in real-time: the ellipsometric response of the metasurface was measured versus time, while temperature was varied by steps in a controlled way using the heating stage. The laser heating experiments were done by shining a continuous violet laser light (Ekspla, $13 \mathrm{~mW}, 409 \mathrm{~nm}$ ) at near-normal incidence on the "cover" metasurface. The ellipsometric response was measured prior to shining the laser, with the laser on and then with the laser off again, each time after waiting that the metasurface temperature was stabilized. In all the temperature-dependent experiments, which were performed in quasidarkness conditions where the reflected light intensity is very low, acquisition times of aprox $20 \mathrm{~s}$ were used for the measurement of $\Delta$ at each photon energy. The optical data analysis and simulations were done with the WVASE32 software (Woollam Co. Inc.); more details are given in Supporting Information S3. 


\section{Supporting Information}

S1. Surface topography of the "cover" metasurface; S2. Room temperature reflectance and ellipsometry measurements on the "uncover" and "cover" metasurfaces; S3. Modeling of the ellipsometry spectra of the "cover" metasurface; S4. Plasmon resonances of the "uncover" and "cover" metasurfaces; S5. Origin of the quasi-darkness conditions in the "cover" metasurface: Thin film interference - plasmon resonance hybridization; S6. Reflectance and ellipsometry measurements versus temperature, and reversibility of the reflected light phase variation upon heating-cooling cycles; S7. Effect of the spectral bandwidth of the incident light on the reflected light phase jump.

\section{Acknowledgements}

The authors are grateful to Thomas Wagner (Lot Oriel Co.) for advice during the data analysis. M.G.P acknowledges support from the European Social Fund and G.B. a CSIC-JAE predoctoral grant, co-funded by the European Social Fund. This work was partially funded by the Spanish Ministry for Economy and Competitiveness through the project MINECO/FEDER TEC2015-69916-C2-1-R.

\section{Author Contribution}

G.B. fabricated the metasurfaces and coordinated the structural characterization under the supervision of J.G. and performed the optical measurements at room temperature; M.G.P. performed the optical measurements as a function of temperature under the supervision of R.S., who designed the experiment; J.T. proposed the concept, performed the optical modeling, fitting and analysis, and wrote the paper with the input from all the authors.

\section{References}

[1] K.D. Heylman, K.A. Knapper, E.H. Horak, M.T. Rea, S.K. Vanga, R.H. Goldsmith, Adv. Mater. 2017, 29, 1700037.

[2] K.D. Heylman, N. Thakkar, E.H. Horak, S.C. Quillin, C. Cherqui, K.A. Knapper, D.J. Masiello, R.H. Goldsmith, Nature Photon. 2016, 10, 788-796.

[3] H.-T. Kim, M. Yu, Opt. Expr. 2016, 24(9), 9501-9510.

[4] L. Stern, A. Naiman, G. Keinan, N. Mzurski, M. Grajower, U. Levy, Optica 2017, $4(1)$. 
[5] L. Wan, H. Chandrahalim, C. Chen, Q. Chen, T. Mei, Y. Oki, N. Nishimura, L.-J. Guo, X. Fan, Appl. Phys. Lett. 2017, 111, 061109.

[6] W. Weng, J.D. Anstie, A.N. Luiten, Phys. Rev. Appl. 2015, 3, 044015.

[7] C.-H. Dong, L. He, Y.-F. Xiao, V.R. Gaddam, S.K. Ozdemir, Z.-F. Han, G.-C. Guo, L. Yang, Appl. Phys. Lett. 2009, 94, 231119.

[8] L. Xu, X. Jiang, G. Zhao, D. Ma, H. Tao, Z. Liu, F.G. Omenetto, L. Yang, Opt. Expr. 2016, 24(18), 20825.

[9] J.-F. Tao, H. Cai, Y.-D. Gu, J. Wu, A.-Q. Lu, IEEE Photon. Tech. Lett. 2015, 27(7), 767.

[10] X. Guan, X. Wang, L.H. Frandsen, Opt. Expr. 2016, 24(15), 16349.

[11] C. Langhammer, E.M. Larsson, B. Kasemo, I. Zoric, Nano Lett. 2010, 10, 3629-3638.

[12] H. Reddy, U. Guler, K. Chaudhuri, A. Dutta, A.V. Kildishev, V.M. Shalaev, A. Boltasseva, ACS Photon. 2017, 4, 1083-1091.

[13] L.J. Davis, M. Deutsch, Rev. Sci. Instr. 2010, 81, 114905.

[14] S.K. Özdemir, J. Light. Tech. 2003, 21(3), 805.

[15] H.-P. Chiang, H.-T. Yeh, C.-M. Chen, J.-C. Wu, S.-Y. Su, R. Chang, Y.-J. Wu, D.-P. Tsai, S.-U. Jen, P.-T. Leung, Opt. Commun. 2004, 241, 409-418.

[16] N. Hirayama, Y. Sano, ISA Trans. 2000, 39, 169-173.

[17] P.S. Maji, R. Das, J. Lightwave Tech. 2017, 35(14), 2833-2839.

[18] N. Yu, F. Capasso, Nature Mater. 2014, 13, 139-150.

[19] A.Y. Zhu, A.I. Kuznetsov, B. Luk'Yanchuk, N. Engheta, P. Genevet, Nanophotonics $\mathbf{2 0 1 7}, 6,2$.

[20] A.M. Urbas, Z. Jacob, L. Dal Negro, et al. J. Opt. 2016, 18, 9.

[21] H. Mühlenbernd, P. Georgi, N. Pholchai, L. Huang, G. Li, S. Zhang, T. Zentgraf, ACS Photon. 2016, 3, 124-129. 
[22] A. Karvounis, B. Gholipour, K.F. MacDonald, N.I. Zheludev, Appl. Phys. Lett. 2016, 109, 051103.

[23] W. Lewandowski, M. Fruhnert, J. Mieckzkowski, C. Rockstuhl, E. Górecka, Nature Comm. 2015, 6, 6590 .

[24] T. Lewi, H.A. Evans, N.A. Butakov, J.A. Schuller, Nano Lett. 2017, 17, 3940-3945.

[25] G. Garcia, R. Buonsanti, A. Llordes, E.L. Runnerstrom, A. Bergerud, D.J. Milliron, Adv. Opt. Mater. 2013, 1, 215-220.

[26] J. Park, J.-H. Kang, S.J. Kim, X. Liu, M.L. Brongersma, Nano Lett. 2017, 17, 407-413.

[27] Z. Zhu, P.G. Evans, R.F. Haglund Jr, J.G. Valentine, Nano Lett. 2017, 17, 4881-4485.

[28] A. Woessner, Y. Gao, I. Torre, M.B. Lundeberg, C. Tan, K. Watanabe, T. Taniguchi, R. Hillenbrand, J. Hone, M. Polini, F.H.L. Koppens, Nature Photon. 2017, 11, 421-424.

[29] M.C. Sherrott, P.W. Hon, K.T. Fountaine, J.C. Garcia, S.M. Ponti, V.W. Brar, L.A. Sweatlock, H.A. Atwater, Nano Lett. 2017, 17, 3027-3034.

[30] K. Thyagararajan, R. Sokhoyan, L. Zornberg, H.A. Atwater, Adv. Mater. 2017, 29, 1701044.

[31] Y.-W. Huang, H.W.H. Lee, R. Sokhoyan, R.A. Pala, K. Thyagarajan, S. Han, D.P. Tsai, H.A. Atwater, Nano Lett. 2016, 16, 5319-5325.

[32] R. Yu, V. Pruneri, F.J. Garcia de Abajo, Sci. Rep. 2016, 6, 32144.

[33] R.F. Waters, P.A. Hobson, K.F. MacDonald, N.I. Zheludev, Appl. Phys. Lett. 2015, 107, 081102.

[34] Y. Yang, K. Kelley, E. Sachet, S. Campione, T.S. Luk, J.-P. Maria, M.B. Sinclair, I. Brener, Nature Photon. 2017, 11, 390-396.

[35] M. Clerici, N. Kinsey, C. DeVault, J. Kim, E.G. Carnemolla, L. Caspani, A. Shaltout, D. Faccio, V. Shalaev, A. Boltasseva, M. Ferrera, Nature Commun. 2017, 8, 15829. 
[36] V.G. Kravets, F. Schedin, R. Jalil, L. Britnell, R.V. Gorbachev, D. Ansell, B.

Thackray, K.S. Novoselov, A.K. Geim, A.V. Kabashin, A.N. Grigorenko, Nature Mater. 2013, 12, 304-309.

[37] M. Svedendahl, R. Verre, M. Käll, Light: Sci. Appl. 2014, 3, e220.

[38] H. Song, N. Zhang, J. Duan, Z. Liu, J. Gao, M.H. Singer, D. Ji, A.R. Cheney, X. Zeng, B. Chen, S. Jiang, Q. Gan, Adv. Opt. Mater. 2017, 5, 1700166.

[39] R. Paniagua-Domínguez, et al. Nature Commun. 2016, 7, 10362.

[40] J. Toudert, X. Wang, C. Tallet, P. Barois, A. Aradian, V. Ponsinet, ACS Photon. 2015, $2,1443-1450$.

[41] A.I. Aristov, M. Manousidaki, A. Danilov, K. Terzaki, C. Fotakis, M. Farsari, A.V. Kabashin, Sci. Rep. 2016, 6, 25380.

[42] L. Malassis, P. Massé, M. Tréguer-Delapierre, S. Mornet, P. Weisbecker, P. Barois, C.R. Simovski, V.G. Kravets, A.N. Grigorenko, Adv. Mater. 2014, 26, 324-330.

[43] A.N. Grigorenko, P.I. Nikitin, A.V. Kabashin, Appl. Phys. Lett. 1999, 75, 3917.

[44] Y. Tsurimaki, J.K. Tong, V.N. Boriskin, A. Semenov, M.I. Ayzatsky, Y.P. Machekhin, G. Chen, S. Boriskina, ACS Photon. 2018.

[45] G. Baraldi, M. Carrada, J. Toudert, F.J. Ferrer, A. Arbouet, V. Paillard, J. Gonzalo, J. Phys. Chem. C 2013, 117 (18), 9431-9439.

[46] J. Toudert, R. Serna, M. Jiménez de Castro, J. Phys. Chem. C 2012, 116 (38), 2053020539.

[47] W. Ahn, S.V. Boriskina, Y. Hing, B.M. Reinhard, ACS Nano 2012, 6(1), 951-960.

[48] M. Barth, S. Schietinger, S. Fischer, J. Becker, N. Nüsse, T. Aichele, B. Löchel, C. Sönnichsen, O. Benson, Nano Lett. 2010, 10, 891-895.

[49] V.R. Dantham, S. Holler, C. Barbre, D. Keng, V. Kolchenko, S. Arnold, Nano Lett. 2013, 13, 3347-3351. 
[50] Y. Luo, M. Chamanzar, A. Apuzzo, R. Salas-Montiel, K. Ngoc Nguyen, S. Blaize, A. Adibi, Nano Lett. 2015, 15, 849-856.

[51] X. Wang, R. Morea, J. Gonzalo, B. Palpant, Nano Lett. 2015, 15, 2633-2639.

[52] D. Jaque, F. Vetrone, Nanoscale 2012, 4, 4301-4326.

[53] S.S. Laha, A.R. Naik, E.R. Kuhn, M. Alvarez, A. Sujkowski, R.J. Wessells, B.P. Jena, Nano Lett. 2017, 17, 1262-1268. 

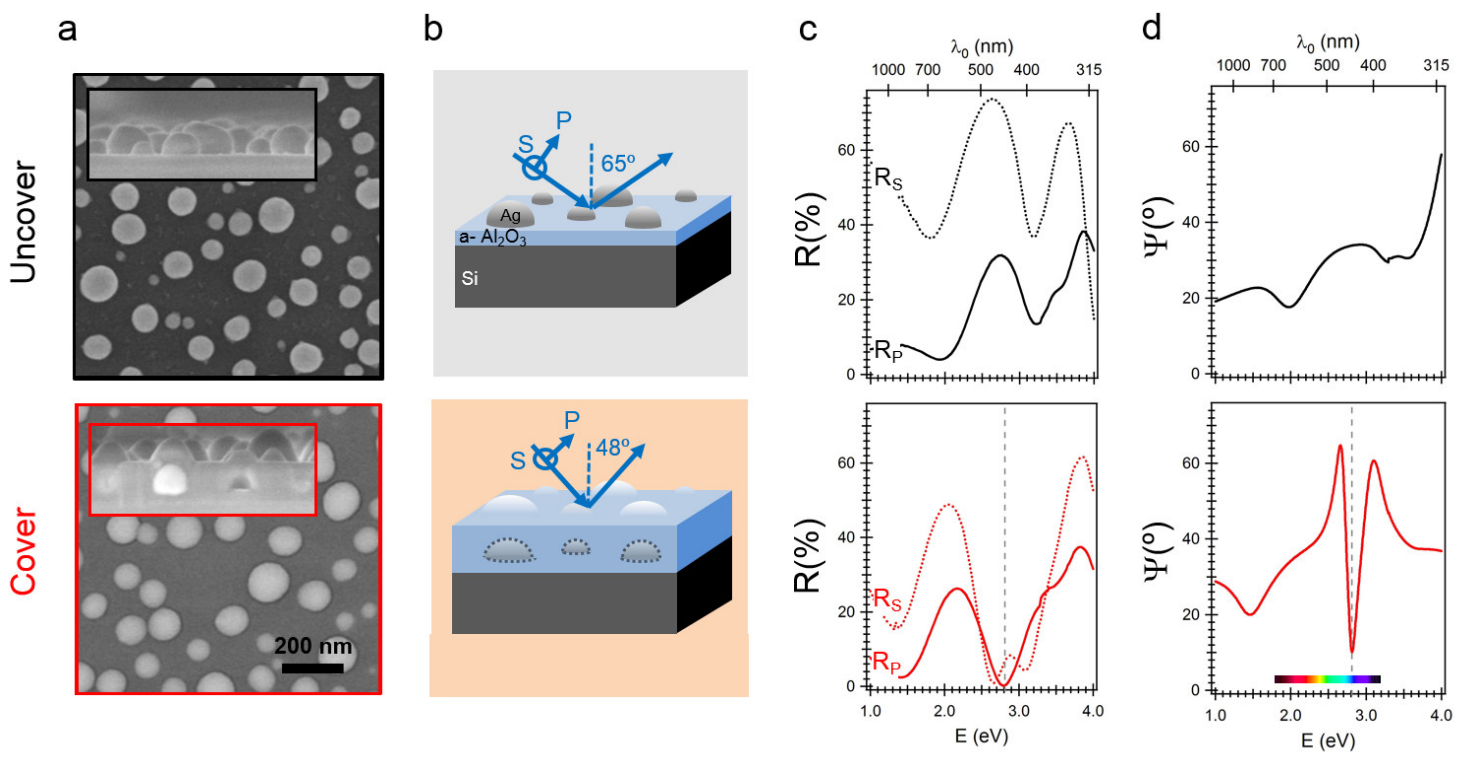

Figure 1. Nanostructure and room-temperature optical properties of the "uncover" and "cover" metasurfaces. (a) Scanning electron images: top-view and cross-section (insets). (b) Configuration of the optical measurements. The orientation of the electric field for $\mathrm{s}-$ and $\mathrm{p}$ polarized incident light is denoted as "s" and "p", respectively. (c) Room-temperature specular reflectance spectra for $s-$ and $p$ - polarized light, $R_{s}$ and $R_{p}$, respectively, for an angle of incidence (AOI) of $65^{\circ}$ ("uncover" metasurface) and $48^{\circ}$ ("cover" metasurface). (d) Roomtemperature spectra of the ellipsometric amplitude coefficient $\Psi$ at the same AOIs. The "cover" metasurface shows a sharp drop and near cancelation of $\Psi$ for (AOI $=48^{\circ}$, photon energy $\mathrm{E} \approx 2.8 \mathrm{eV}$ ) where quasi-darkness conditions are fulfilled. 

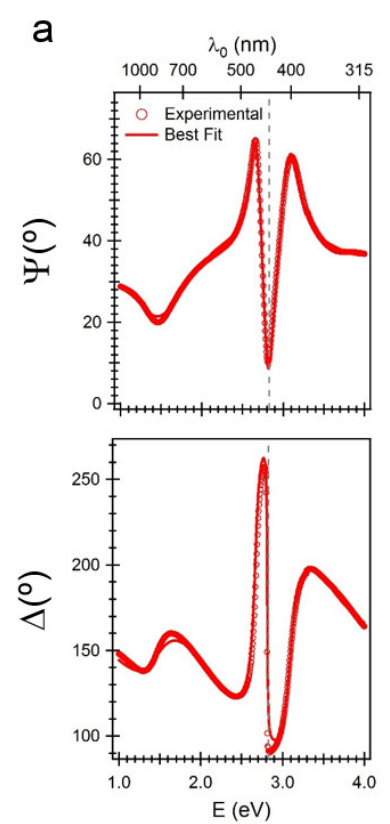

b

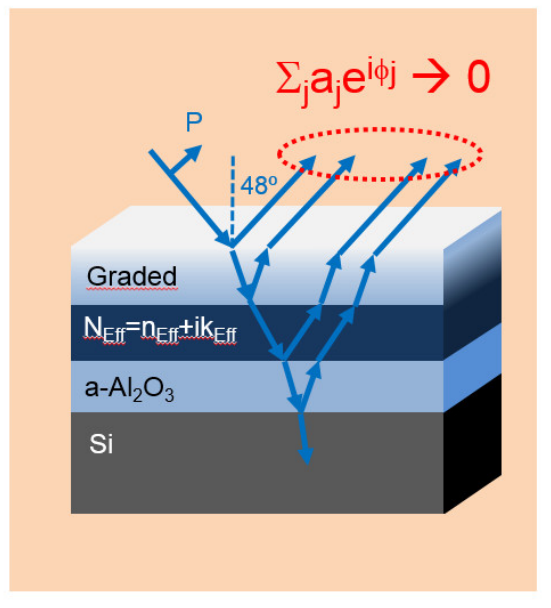

C
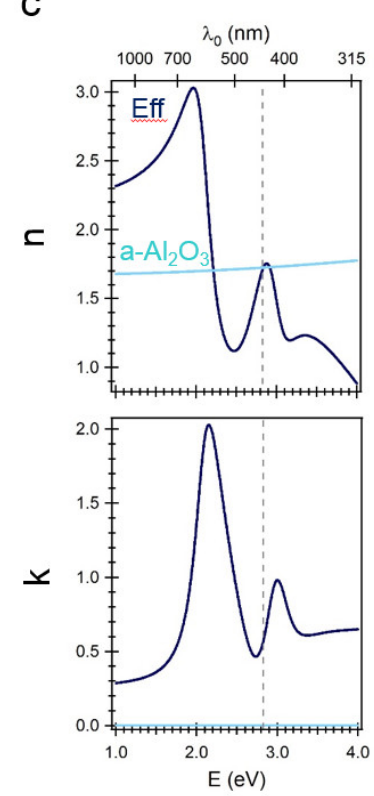

Figure 2. "Cover" metasurface: reflected light phase jump in quasi-darkness conditions enabled by thin film interference - plasmon resonances hybridization. (a) Experimental and best-fit simulated room-temperature spectra of the ellipsometric amplitude and phase coefficients, $\Psi$ and $\Delta$, for an AOI of $48^{\circ}$. An abrupt $\Delta$ variation occurs in the quasi-darkness conditions (AOI $=48^{\circ}, \mathrm{E} \approx 2.8 \mathrm{eV}$ ). (b) Equivalent model (layered structure) used for simulating the ellipsometry spectra. The buried Ag nanoparticles layer is represented by an effective medium with complex refractive index $\mathrm{N}_{\text {eff }}=n_{\text {eff }}+i k_{\text {eff. }}$ (c) Best-fit spectra of $n_{\text {eff }}$ and $\mathrm{k}_{\text {eff }}$ together with the $\mathrm{n}$ and $\mathrm{k}$ of $\mathrm{a}-\mathrm{Al}_{2} \mathrm{O}_{3}$. The vertical dashed line points at $\mathrm{E} \approx 2.8 \mathrm{eV}$ where the quasi-darkness conditions are fulfilled (for an AOI of $48^{\circ}$ ). The specific values of $\mathrm{n}_{\text {eff }}$ and $\mathrm{k}_{\mathrm{eff}}$ at this $\mathrm{E}$ value allow almost fully destructive interferences for the p-polarized reflected light, as depicted in red in (b). 

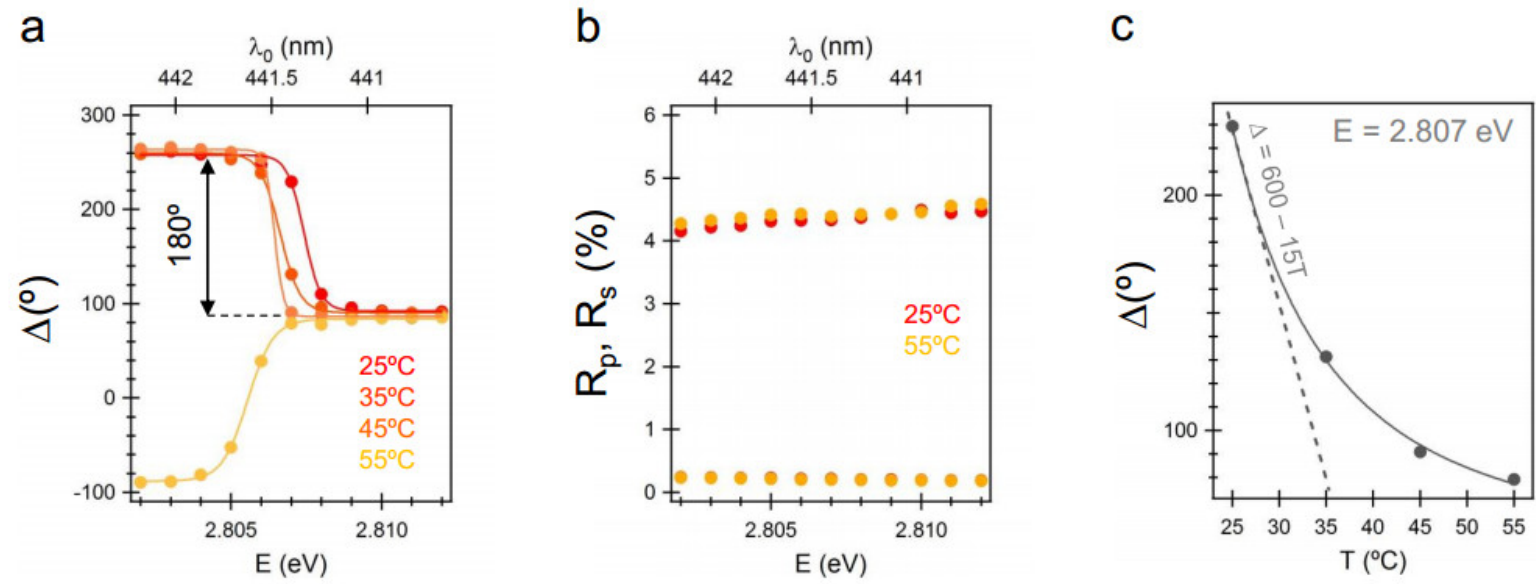

d
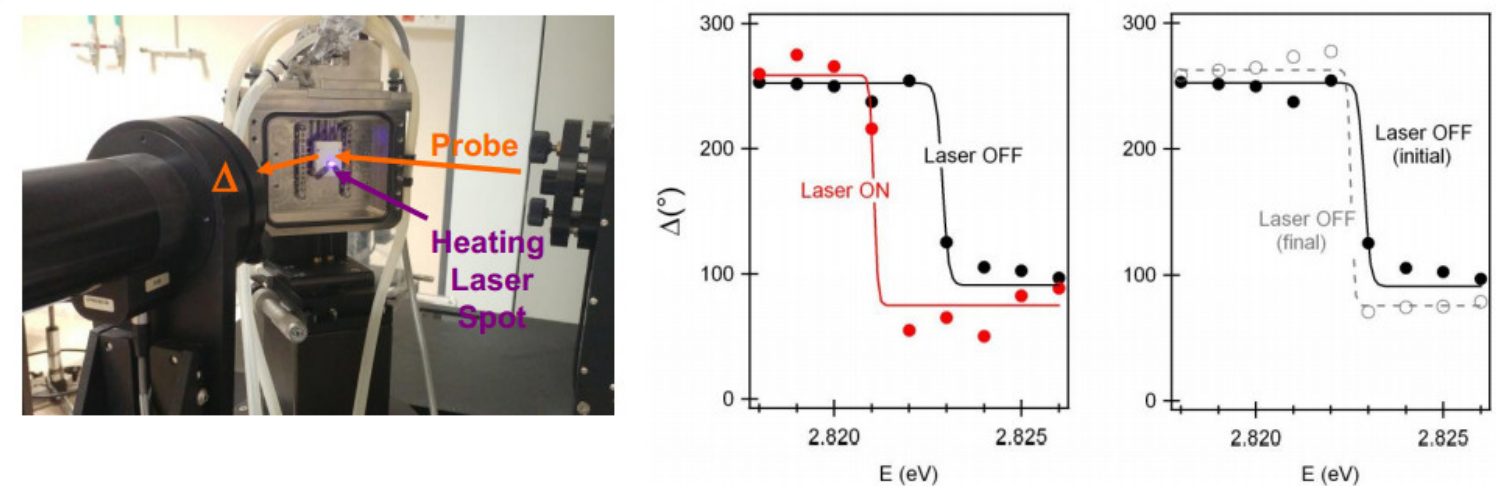

Figure 3. Optical thermometry by monitoring the phase of the reflected light in quasidarkness conditions. (a) Spectrum of the ellipsometric coefficient $\Delta$ of the "cover" metasurface (AOI $=48^{\circ}$ ) at different temperatures from $25^{\circ} \mathrm{C}$ to $55^{\circ} \mathrm{C}$. (b) Specular reflectance spectra for s- polarized and p- polarized light $\left(R_{s}\right.$ and $\left.R_{p}\right)$ of the same metasurface at $25^{\circ} \mathrm{C}$ and $55^{\circ} \mathrm{C}$. (c) Variation of the ellipsometric coefficient $\Delta$ at a photon energy of $2.807 \mathrm{eV}$ as a function of temperature. The dashed line is a linear fit of the low temperature part of the graph, yielding the responsivity of the optical thermometry scheme: $15^{\circ}$ in $\Delta$ per ${ }^{\circ} \mathrm{C}$ in temperature. (d) Optical thermometry during laser heating of a "cover" metasurface: picture of the experimental setup (left), spectrum of the ellipsometric coefficient $\Delta$ before shining the laser vs with the laser on (middle), and before shining the laser and after turning it off (right). This shows the suitability of the metasurface for monitoring optically laser-induced heating, and the reversibility of the measurement scheme. 
A self-assembled nanostructured metasurface that enables practical high-resolution optical thermometry is presented. It combines, in a subwavelength thickness, thin film interference with low-quality-factor plasmon resonances to achieve a quasi-darkness response with a sharp spectral jump of the optical phase. Such jump is equivalent with a high-qualityfactor resonator that yields a high sensor resolution with a facile, yet powerful, experimental scheme.

\section{Keyword: Optical Metasurface Sensors}

Giorgio Baraldi, Marina García Pardo, José Gonzalo, Rosalia Serna*, Johann Toudert*

Self-assembled nanostructured photonic-plasmonic metasurfaces for high-resolution optical thermometry
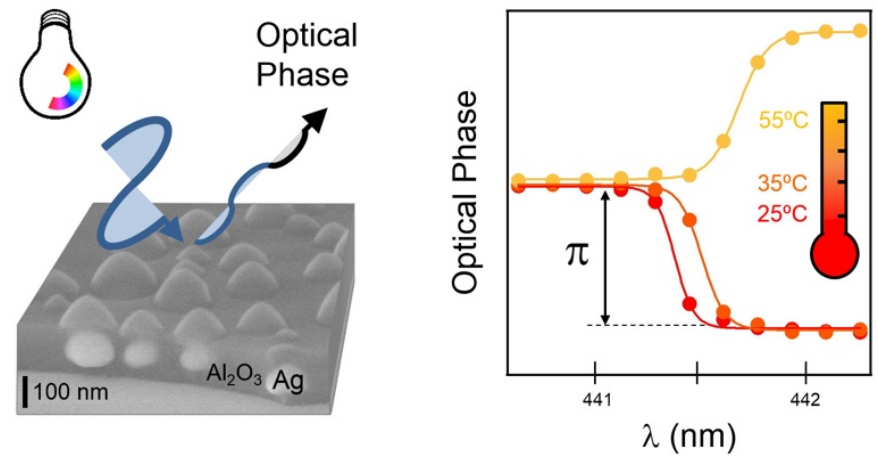
Copyright WILEY-VCH Verlag GmbH \& Co. KGaA, 69469 Weinheim, Germany, 2016.

Supporting Information

Self-assembled nanostructured photonic-plasmonic metasurfaces for high-resolution optical thermometry

Giorgio Baraldi, Marina García Pardo, José Gonzalo, Rosalia Serna*, Johann Toudert* 


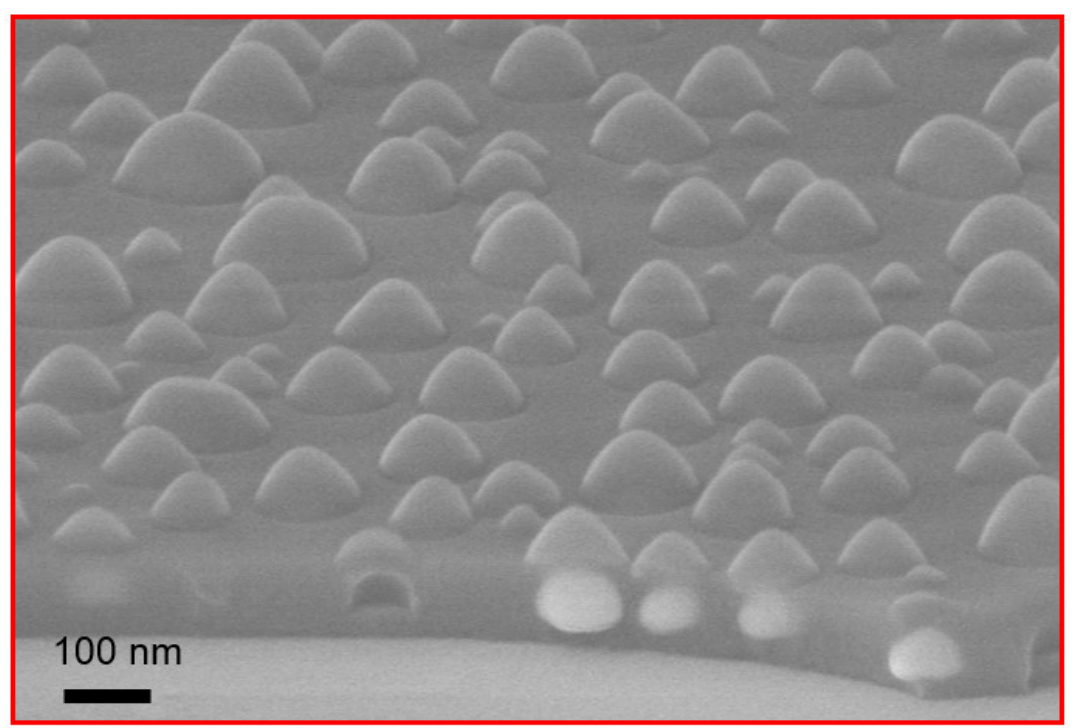

Figure S1.1. Tilted view scanning electron microscope image of the "cover" metasurface showing that the amorphous array of $\mathrm{a}-\mathrm{Al}_{2} \mathrm{O}_{3}$ nanodomes replicates conformally the morphology and distribution of the nanoparticles underneath.

\section{S2. Room temperature reflectance and ellipsometry measurements on the "uncover" and "cover" metasurfaces}

Room temperature specular reflectance spectra were measured with a Woollam VASE ellipsometer (Woollam Co. Inc.) set in photometry mode. Light was generated by a white light source (Xe lamp), filtered spectrally by a single monochromator. The monochromated light was collimated and passed through a polarizer which orientation was chosen to generate the desired linear polarization ( $\mathrm{s}-$ or $\mathrm{p}$-) for the light impinging onto the sample. The reflected light was detected in a specular reflectance configuration (angle of detection equal to the angle of incidence) by a silicon detector. The specular reflectance $R_{p}$ and $R_{s}$ were then determined at each photon energy by dividing the reflected intensity by that of the white light source.

Room temperature ellipsometry spectra were measured with the same ellipsometer in a PRSA $_{R}$ configuration. This time, the monochromated and collimated light passed through a Polarizer-Retarder setup before impinging on the Sample. The specularly reflected light was analyzed by a Rotating Analyzer before being collected by the silicon detector. The dynamic averaging option was activated during all the measurements. The monochromator slit width was set in automatic mode. In the spectral region of the "covered" metasurface quasi-darkness conditions, the intensity reflected by the sample for the corresponding polarization is very low, 
and thus the slits are opened at the maximum $(1.7 \mu \mathrm{m})$ In these conditions, the spectral bandwidth of the light impinging onto the sample for a given nominal photon energy amounts to $8 \mathrm{~nm}$.
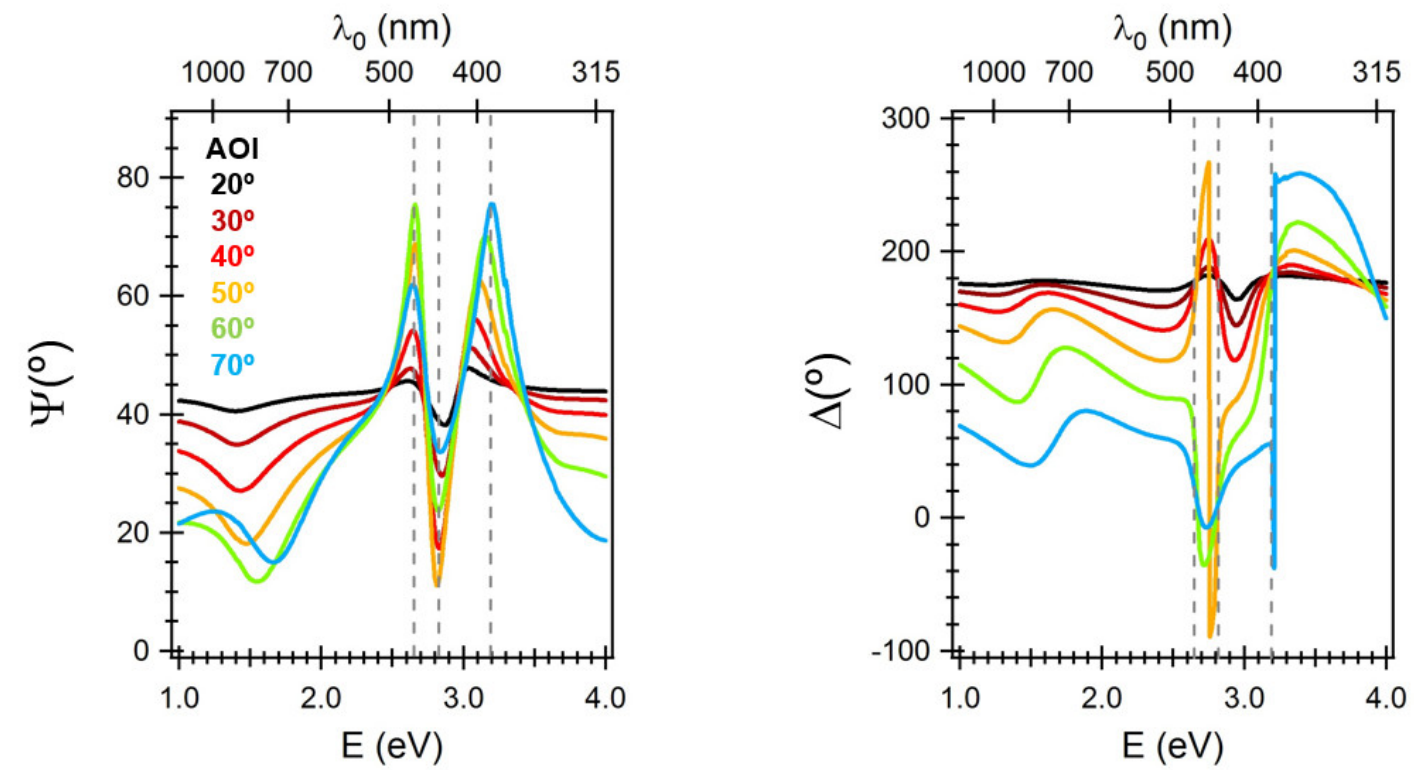

Figure S2.1. Room temperature experimental spectra of the ellipsometric amplitude and phase coefficients, $\Psi$ and $\Delta$, of the "cover" metasurface at several AOIs.
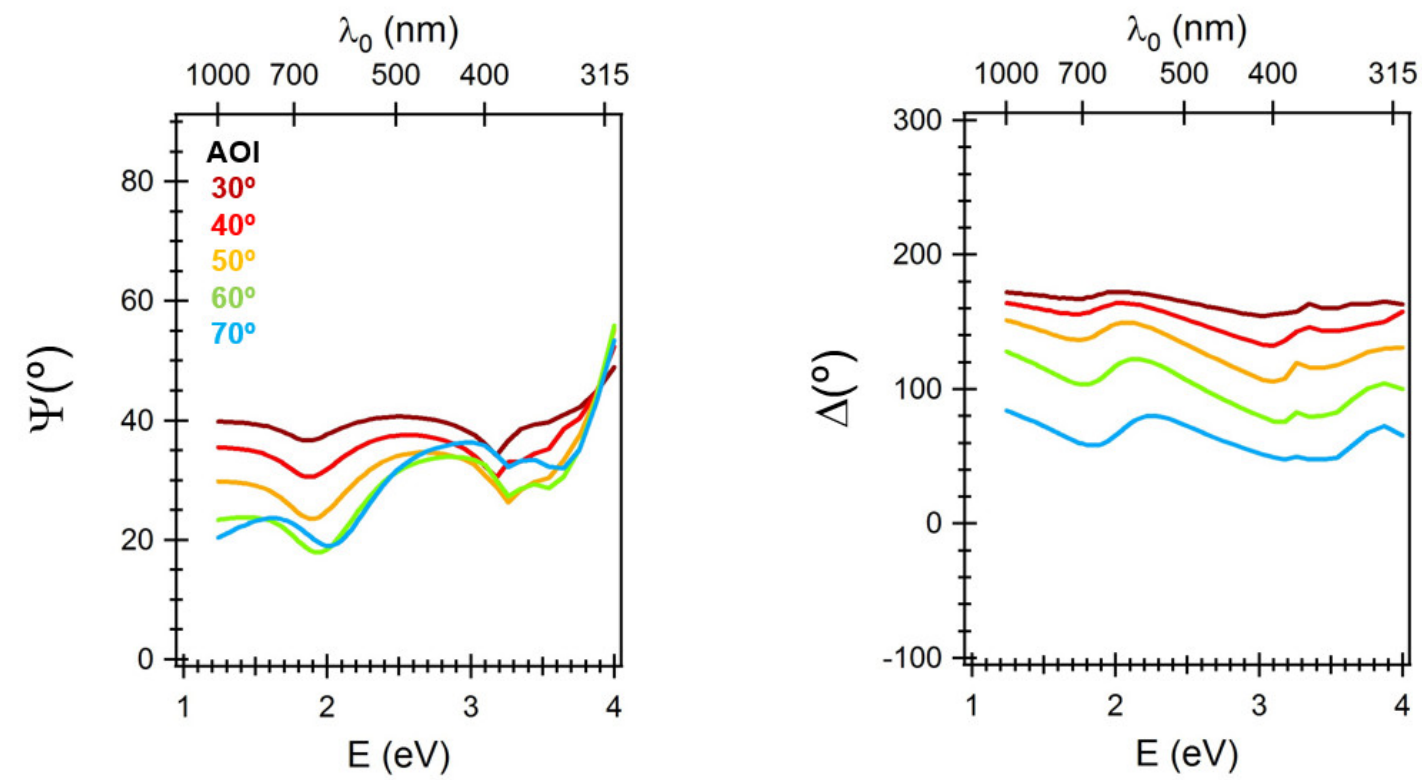

Figure S2.2. Room temperature experimental spectra of the ellipsometric amplitude and phase coefficients, $\Psi$ and $\Delta$, of the "uncover" metasurface at several AOIs. 


\section{S3. Modeling of the ellipsometry spectra of the "cover" metasurface}

\section{Description of the model}

The simulation and fitting of the $\Psi$ and $\Delta$ spectra were performed with the WVASE32 software (Woollam Co. Inc.). The "cover" metasurface was modeled as a multilayer system, where the layer of buried Ag nanoparticles was represented by an effective medium with refractive index $\mathrm{N}_{\text {eff }}=\mathrm{n}_{\text {eff }}+\mathrm{ik}_{\mathrm{eff}}$, and the conformal a- $\mathrm{Al}_{2} \mathrm{O}_{3}$ cover layer was modeled as a graded medium. The dielectric function $\varepsilon_{\text {eff }}=\left(\mathrm{N}_{\mathrm{eff}}\right)^{2}$ of the effective medium was modeled as the sum of a constant term and Kramers-Kronig consistent Lorentz oscillators (Generalized Oscillator model). The graded medium was modeled as a stack of Bruggeman EMA a$\mathrm{Al}_{2} \mathrm{O}_{3}$ :void layers with the volume fraction of void increasing from the bottom to the top layer, covered by an EMA roughness layer. The model structure is depicted in Figure S3.1. Note that incoherent effects were taken into account in the simulation by including the thickness non-uniformity and the finite spectral bandwidth of the incident light (related with the monochromator aperture) as additional parameters.

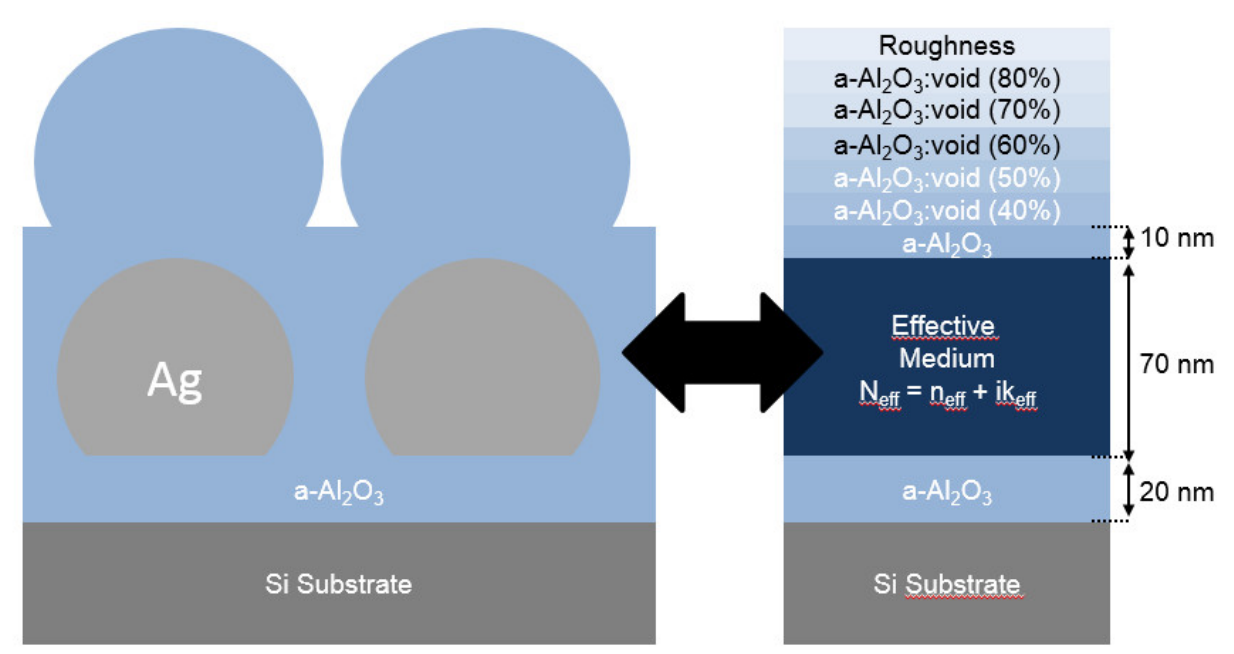

Figure S3.1. Structure used for the modelling of the ellipsometry spectra for the "cover" metasurface.

Using this model, the measured $\Psi$ and $\Delta$ spectra at $48^{\circ}$ were fitted, the free parameters being those of the effective layer (constant term and energy, width, amplitude of the Lorentz oscillators) and thickness non-uniformity. The dielectric function of a- $\mathrm{Al}_{2} \mathrm{O}_{3}$ was determined previously by spectroscopic ellipsometry on pure a- $\mathrm{Al}_{2} \mathrm{O}_{3}$ films, and fixed at this value (see Figure 2c) in the present fitting procedure. The layer thicknesses were fixed at values extracted from the scanning electron microscope cross-section images, and the spectral 
bandwidth of the incident light was fixed at $8 \mathrm{~nm}$, its actual value for the maximum aperture of the monochromator slits. The best-fit thickness non-uniformity amounted to $18 \%$. Note that this value, which plays a significant role only in the spectral region of the phase jump, does likely not account for a real non-uniformity of the films that were homogeneous at the centimeter scale. In contrast, it might account for incoherent effects introduced by light scattering that cannot be modeled explicitly using the WVASE software.

\section{Validity of the modeling approach}

It is often considered that, for effective medium modeling to be valid and meaningful for the description of the optical properties of a metamaterial, the structures constituting such material much be much smaller than the wavelength of the incident light. However, many examples show that this condition is sometimes not sufficient, and sometimes too strict. ${ }^{[s 1-s 6]}$ Therefore, to know if effective medium modeling can be applied to a given metamaterial, a practical approach consists in building a model capturing its main structural and physical features and looking how accurately it can reproduce the measured optical properties. ${ }^{[\mathrm{s} 1-\mathrm{s} 6]}$ It is the approach followed in this work. We show that indeed there is an excellent agreement between the calculation with the effective medium model and the experiment. This agreement is shown in Fig. 2a, where the experimental and simulated spectra overlap.

In Fig. S3.2, we give further proof of the validity of this model. It is seen that it enables fitting simultaneously the $\Psi, \Delta, \mathrm{R}_{\mathrm{p}}$ and $\mathrm{R}_{\mathrm{s}}$ spectra of the "cover" metasurface in a broad range of photon energies $(1-3.3 \mathrm{eV}$, that includes the two plasmon resonance modes) and a broad range of AOIs (from $20^{\circ}$ to $70^{\circ}$ ). As seen in Fig. S3.3, the effective $\mathrm{N}_{\text {eff }}$ obtained by the simultaneous fitting of all the $\Psi, \Delta, R_{p}$ and $R_{s}$ spectra at all angles is very similar to that obtained by fitting at a single AOI $\left(48^{\circ}\right)$ the $\Psi$ and $\Delta$ spectra only, and shows two resonance modes that agree with the transmittance spectra of Fig. S4.1.

Achieving such multi-angle and multi-spectra fitting with a single model is a remarkable fact and a solid evidence that using the effective medium approximation is valid for reproducing the experimental spectra of the "cover" metasurface and capturing its physical features. 

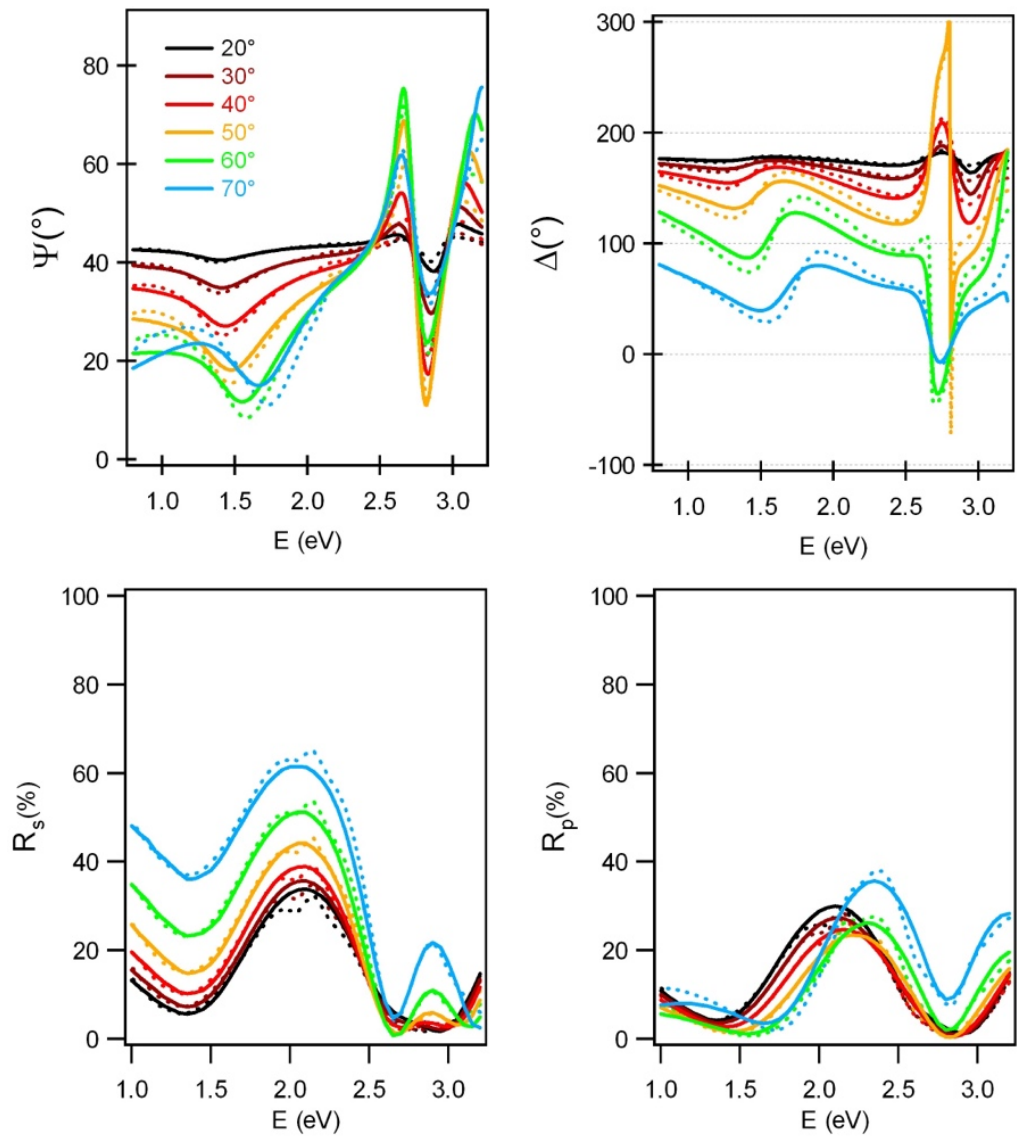

Figure S3.2. Experimental (continuous lines) and fitted (dashed lines) $\Psi, \Delta, \mathrm{R}_{\mathrm{p}}$ and $\mathrm{R}_{\mathrm{s}}$ spectra of the "cover" metasurface. The fitted curves were obtained by fitting simultaneously all the spectra with a single model, that is shown in Fig S3.1. The corresponding best fit $\mathrm{N}_{\text {eff }}$ spectrum is shown in Fig. S3.3.

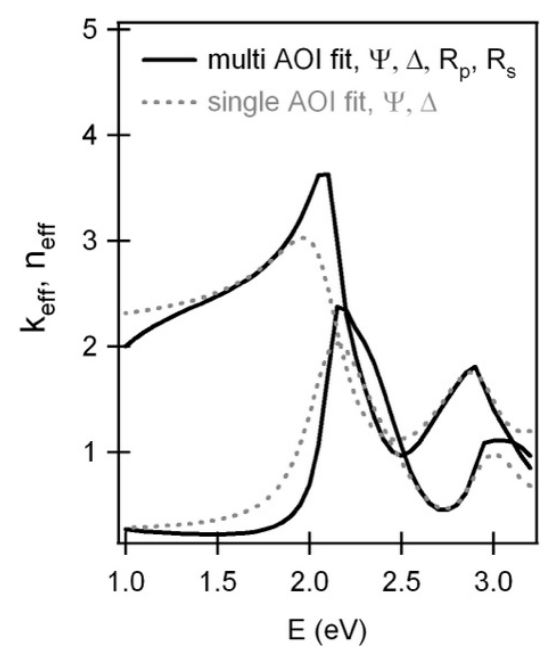

Figure S3.3. Best fit $\mathrm{N}_{\text {eff }}$ spectrum ( $\left.\mathrm{N}_{\text {eff }}=\mathrm{n}_{\text {eff }}+\mathrm{i} \mathrm{k}_{\text {eff }}\right)$ obtained from the simultaneous fitting of the $\Psi, \Delta, \mathrm{R}_{\mathrm{p}}$ and $\mathrm{R}_{\mathrm{s}}$ spectra of the "cover" metasurface for AOIs from $20^{\circ}$ to $70^{\circ}$ by steps of $10^{\circ}$, using a single model, that is described in Fig. S3.1. 


\section{S4. Plasmon resonances of the "uncover" and "cover" metasurfaces}

The room temperature transmittance spectra of the "uncover" and "cover" metasurfaces grown on a transparent fused silica substrate were measured at normal incidence using a double-beam Varian Cary 5000 spectrophotometer. A pure a- $\mathrm{Al}_{2} \mathrm{O}_{3}$ film was also measured, showing the excellent transparency of this material in the spectral range of the experiment.

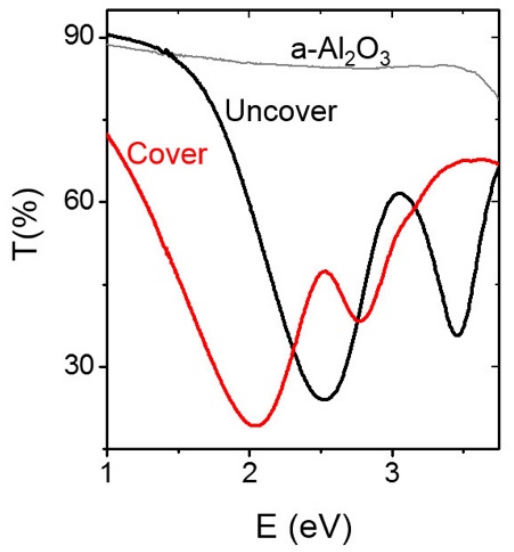

Figure S4.1. Room temperature transmittance spectra of the "uncover" and "cover" metasurfaces on a transparent fused silica substrate. The two absorption bands seen for each are attributed to plasmon resonance modes, dipole and quadrupole ${ }^{[s]}$ The transmittance spectrum of an $\mathrm{a}-\mathrm{Al}_{2} \mathrm{O}_{3}$ film is shown for comparison.

To evidence the role of the resonances on the reflectance spectra of the "cover" metasurface on Si substrate, simplified FDTD simulations were performed. A monodisperse, perfectly organized array of Ag nanoparticles with shape/size characteristic was considered. For the nanoparticle size, shape, density and $\mathrm{a}-\mathrm{Al}_{2} \mathrm{O}_{3}$ layer thickness considered, a maximum reflectance is found near the photon energy of $1.45 \mathrm{eV}$, where a strong dipolar resonance occurs thus radiating to the far-field.

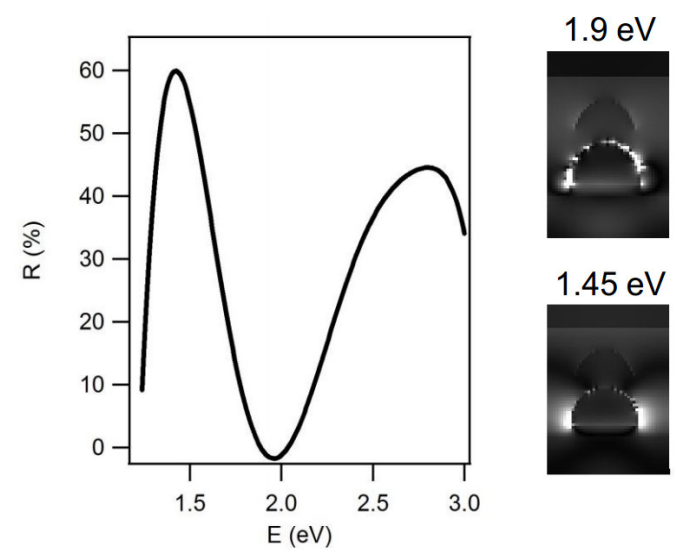

Figure S4.2. FDTD simulations of an array of $\mathrm{Ag}$ nanoparticles covered with a- $\mathrm{Al}_{2} \mathrm{O}_{3}$, on a $\mathrm{Si}$ substrate (in plane nanoparticle diameter $120 \mathrm{~nm}$, center to center distance $240 \mathrm{~nm}$ ). Left: smoothed simulated reflectance at normal incidence. Right: maps of the electric field intensity at photon energies of $1.9 \mathrm{eV}$ and $1.45 \mathrm{eV}$. 


\section{S5. Origin of the quasi-darkness conditions in the "cover" metasurface: Thin film interference - plasmon resonance hybridization}

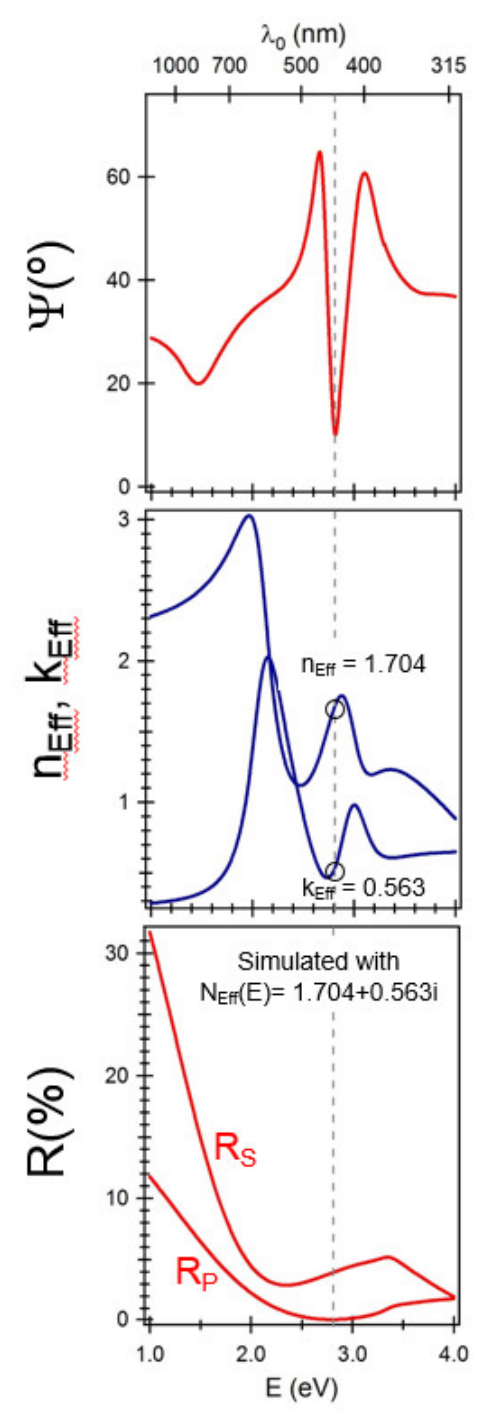

Figure S5.1. Top: Room-temperature spectrum of the ellipsometric amplitude coefficient $\Psi$ of the "cover" metasurface $\left(\mathrm{AOI}=48^{\circ}\right)$. Middle: Best-fit room-temperature spectra of the refractive index $n_{\text {eff }}$ and extinction coefficient keff of the effective layer accounting for the embedded Ag nanoparticles in the "cover" metasurface $\left(A O I=48^{\circ}\right)$. Bottom: Spectra of the s-polarized and p-polarized reflectance spectra $\left(R_{s}\right.$ and $\left.R_{p}, A O I=48^{\circ}\right)$ simulated for a structure identical to that of the "cover" metasurface, but assuming photon energy - independent $n_{\text {eff }}$ and $k_{\text {eff }}$ for the effective layer $\left(n_{\text {eff }}=1.704\right.$ and $k_{\text {eff }}=0.563$ ). These values are those found by the fitting procedure for the "cover" metasurface at the photon energy of its quasi-darkness conditions for p-polarized light (minimum of $\Psi$ ).

In order to investigate the origin of the quasi-darkness conditions of the "cover" metasurface, we performed an analysis similar to that done in Ref. 40. The spectra of the s- and p-polarized reflectance $\left(R_{s}\right.$ and $\left.R_{p}, A O I=48^{\circ}\right)$ of a structure identical to that of the "cover" metasurface (Figure S3.1) were simulated, replacing the best fit $\mathrm{n}_{\text {eff }}$ and $\mathrm{k}_{\mathrm{eff}}$ spectra (shown in Figure S5.1, 
middle) by wavelength-independent $n_{\text {eff }}$ and $k_{\text {eff: }} n_{\text {eff }}=1.704$ and $k_{\text {eff }}=0.563$. These values are those found by the fitting procedure for the "cover" metasurface at its quasi-darkness conditions for $\mathrm{p}$-polarized light $\left(\mathrm{AOI}=48^{\circ}, \mathrm{E} \approx 2.8 \mathrm{eV}\right.$ ). It is seen that the simulated $\mathrm{R}_{\mathrm{p}}$ spectrum reaches a minimum near-zero value at $\mathrm{E} \approx 2.8 \mathrm{eV}$ only, while $\mathrm{R}_{\mathrm{s}}$ remains above $4 \%$ in the whole spectral range considered. This proves that the quasi-darkness conditions found for the "cover" metasurface result from destructive interference in the metasurface structure. Since this destructive interference occurs at specific $\left(\mathrm{n}_{\text {eff }}, \mathrm{k}_{\mathrm{eff}}\right)$ values imposed by the plasmon resonances of the Ag nanoparticles, it is concluded that the quasi-darkness conditions result from the hybridization between thin film interference and plasmon resonances, i.e. a process involving optical interference modulated spectrally by plasmonic effects. 


\section{S6. Reflectance and ellipsometry measurements versus temperature, and reversibility of the reflected light phase change upon heating-cooling cycles}

The ellipsometric phase coefficient $\Delta$ and the s- polarized and p-polarized reflectance of the "cover" metasurface were measured as a function of temperature in the $25^{\circ} \mathrm{C}-55^{\circ} \mathrm{C}$ range. A commercial, computer-controlled heating stage (Instec Co.) installed on the VASE ellipsometer was used to set the temperature at the desired value and maintain it during the optical measurement. In all the experiments with this setup, the temperature was varied by steps in a controlled way with the heating stage, and the ellipsometric response was measured at selected times, corresponding to selected temperatures. Figure S6.1 shows the data as they are obtained in our experiments, where the evolution of $\Delta$ and the temperature can be represented as a function of time.

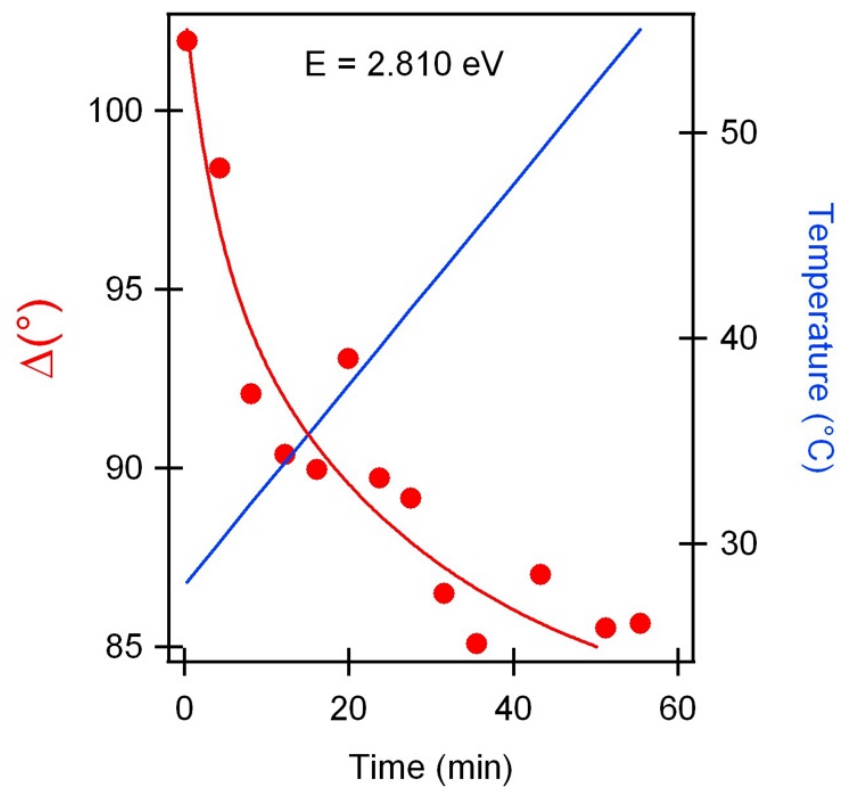

Figure S6.1. Example showing the data obtained during the temperature-dependent ellipsometry experiments with the "cover" metasurface, for a given photon energy. Temperature is varied linearly by small steps and $\Delta$ is measured at selected times corresponding to different temperatures.

The measurements were restricted to the photon energy range from 2.80 to $2.82 \mathrm{eV}$ where the quasi-darkness induced phase jump occurs and the phase of the near-canceled p-polarized reflected light is highly sensitive to temperature. Figure S6.2 exemplifies such sensitivity at different photon energies. Figure S6.3 illustrates the reversibility of the change in $\Delta$ upon heating-cooling cycles. After heating from $25^{\circ} \mathrm{C}$ to $55^{\circ} \mathrm{C}$ and cooling down to $25^{\circ} \mathrm{C}, \Delta$ comes back to its initial value. 


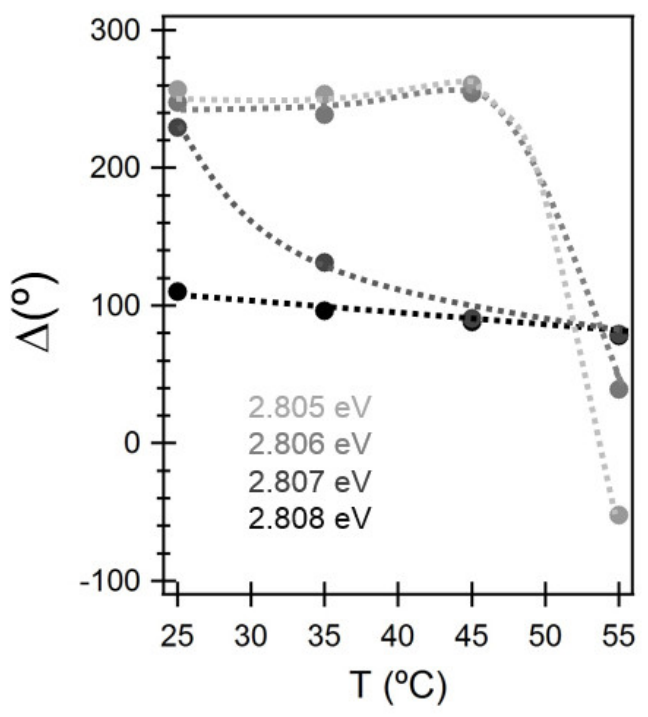

Figure S6.2. Variation of the phase of the near-canceling p-polarized light reflected by the "cover" metasurface as a function of temperature for given photon energies E, as measured from the spectra of the ellipsometric phase coefficient $\Delta$.

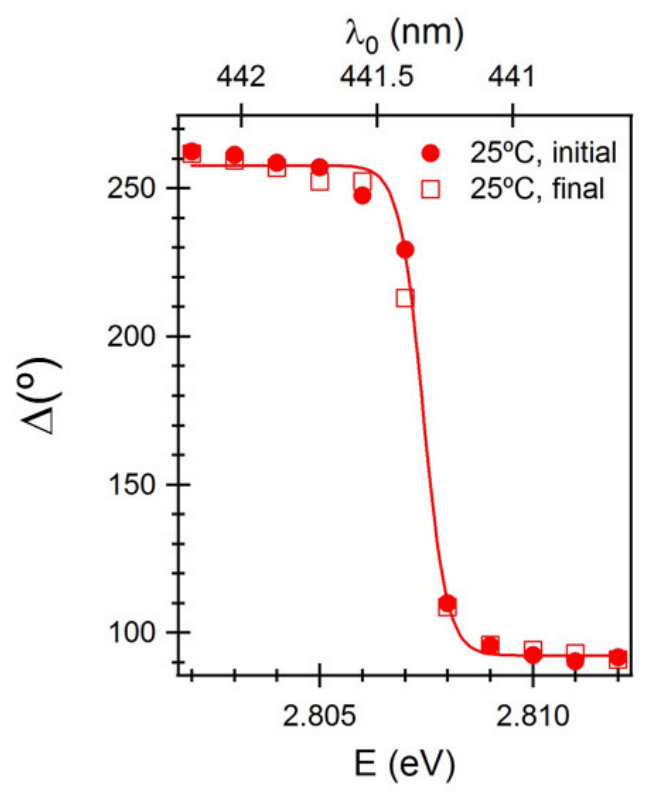

Figure S6.3. Reversibility of the reflected light phase jump of the "cover" metasurface upon heating-cooling cycles, as measured from the spectra of the ellipsometric phase coefficient $\Delta$. The sample was heated from $25^{\circ} \mathrm{C}$ $\left(25^{\circ} \mathrm{C}\right.$, initial) to $55^{\circ} \mathrm{C}$ and cooled down to $25^{\circ} \mathrm{C}\left(25^{\circ} \mathrm{C}\right.$, final).

Note that, because all the measurements performed in quasi-darkness conditions deal with low intensities of reflected light, reaching a good resolution in $\Delta$ (primordial to reach a good temperature resolution) requires suitable measurement conditions to maximize the signal-tonoise ratio. In our experiments, acquisition times of aprox $20 \mathrm{~s}$ per photon energy were used. 


\section{S7. Effect of the spectral bandwidth of the incident light on the reflected light phase jump}

To evaluate the effect of the finite spectral bandwidth of the incident light on the quasidarkness - related reflected light phase jump of the "cover" metasurface at room temperature, simulations were done using the model described in Section S3. All the parameter values were fixed at the best-fit ones found previously, except the spectral bandwidth of the incident light that was varied in the range from 0 to $9 \mathrm{~nm}$. The simulated spectra of the ellipsometric phase coefficient $\Delta$ at an AOI of $48^{\circ}$ and for different spectral bandwidths of the incident light are shown in Figure S7.1. The variation in $\Delta$ with bandwidth is too small to induce any smearing out of the phase jump.

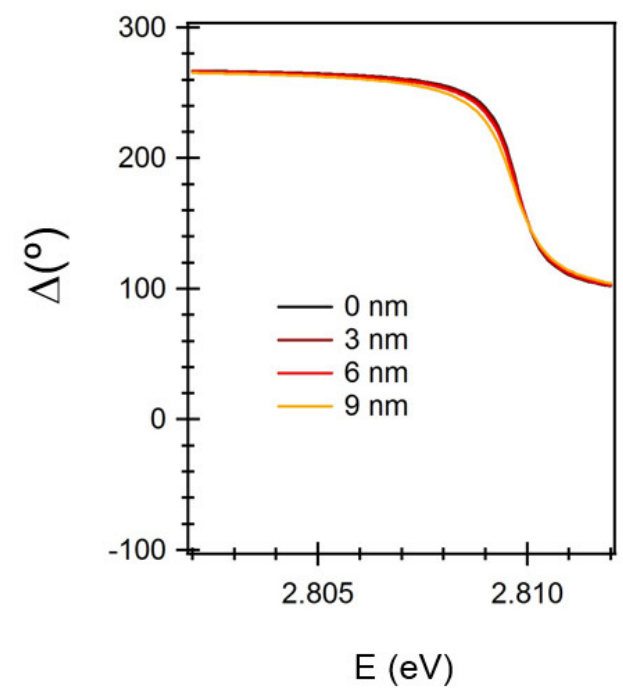

Figure S7.1. Simulated spectra of the ellipsometric coefficient $\Delta$ of the "cover" metasurface around the reflected light phase jump, as a function of the spectral bandwidth of the incident light (room temperature, $\mathrm{AOI}=48^{\circ}$ ). 


\section{Supporting Information - References}

[s1] D.R. Smith, D.C. Vier, Th. Koschny, C.M. Soukoulis, Phys. Rev. E 2005, 71, 036617.

[s2] N.C. Dyck, R. C. Denomme, P.M. Nieva, J. Phys. Chem. C 2011, 115, 15225-15233.

[s3] A. Malasi, R. Kalyanaraman, H. Garcia, J. Opt. 2014, 16, 065001.

[s4] Z. Zhang, Y. Wu, Sci. Rep. 2015, 5, 7892.

[s5] P.T. Bowen, A. Baron, D.R. Smith, Phys. Rev. B 2017, 95, 033822.

[s6] N. Guth, B. Gallas, J. Rivory, J. Grand, A. Ourir, G. Guida, R. Abdeddaim, C. Jouvaud, J. de Rosny, Phys. Rev. B 2012, 85, 115138.

[s7] X. Liu, D. Li, X. Sun, Z. Li, H. Song, H. Jiang, Y. Chen, Sci. Rep. 2015, 5, 12555. 